\title{
An iterative method for the design of variable fractional-order FIR differintegrators
}

\author{
Jong-Jy Shyu ${ }^{\mathrm{a}, *}$, Soo-Chang Pei ${ }^{\mathrm{b}}$, Cheng-Han Chan ${ }^{\mathrm{c}}$ \\ a Department of Electrical Engineering, National University of Kaohsiung, No. 700, Kaohsiung University Rd., Nan-Tzu District, Kaohsiung 811, Taiwan, ROC \\ ${ }^{\mathrm{b}}$ Department of Electrical Engineering, National Taiwan University, Taiwan, ROC \\ c Department of Computer and Communication Engineering, National Kaohsiung First University of Science and Technology, Taiwan, ROC
}

\section{A R T I C L E I N F O}

\section{Article history:}

Received 22 April 2008

Received in revised form

15 September 2008

Accepted 17 September 2008

Available online 4 October 2008

Keywords:

Variable fractional-order differintegrator

FIR digital filter

Digital differentiator

Digital integrator

Weighted-least-squares approach

\begin{abstract}
A B S T R A C T
In the paper, a new method is proposed for the design of variable fractional-order (VFO) FIR differintegrators. Comparing with the existing methods, the elements of relevant matrices can be determined just by the given specification, which makes the method easier. An iterative technique is also incorporated to adjust the weighting function, such that the peak absolute error of variable frequency response can be reduced drastically. Several design examples, including a VFO differintegrator, two VFO differentiators and a VFO integrator, are presented to demonstrate the effectiveness and flexibility of the proposed method.
\end{abstract}

(c) 2008 Elsevier B.V. All rights reserved.

\section{Introduction}

Fractional calculus, which deals with derivatives and integrals of arbitrary order [1-4], is an important topic in mathematical analysis. The theory of fractional-order derivatives and integrals was developed in the seventeenth century. However, just during the last three decades, the concept of fractional calculus has been investigated in different areas of engineering applications such as electromagnetic theory, fluid flow, automatic control, electrical networks and signal processing [5-10].

Recently, several methods have been developed to design digital fractional-order differentiators and integrators including filtering technique, discretization method, frequency-domain approximation, fractional sample delay technique and factorization process [11-19]. Also, there is a branch of trend concerns the design of variable digital

\footnotetext{
* Corresponding author. Tel.: +88675919236; fax: +88675919374.

E-mail addresses: jshyu@nuk.edu.tw (J.-J. Shyu), pei@cc.ee.ntu.edu.tw (S-.C. Pei), u9115902@ccms.nkfust.edu.tw (C.-H. Chan).
}

filters which are applied to where the frequency characteristics need to be adjustable. The variable digital filters are generally classified into two categories. One is the filters with adjustable magnitude response such as the filters with variable cut-off frequencies and the variable fractional-order (VFO) differentiators [20-24]. The other is the filters with variable fractional-delay response [25-29] which are used in music instrument modeling, sampling rate conversion, discrete time signal interpolation and time delay estimation.

In the paper, an iterative method is proposed for the design of VFO FIR differintegrators. The method is originally used to design 2-D FIR digital filters in leastsquares sense, including quadrantally symmetric/antisymmetric 2-D FIR filters and complex coefficient 2-D filters [30-32]. However, in the paper we do not intend to derive the closed forms as in [30-32], and the method is modified by incorporating weighting functions such that it can design VFO FIR differintegrators in weighted-leastsquares sense. Moreover, by applying proper iterative technique [33], the peak absolute error of variable frequency response can be reduced drastically. Comparing 
with the existing methods [21,22], the values of relevant matrices can be obtained just from the given specification, also there is no need of closed-form formulation as the design of variable fractional-delay filters [28,29]. The paper is organized as follows. Section 2 deals with the problem formulation, in which the used transfer function is characterized such that the Farrow structure [26] can be applied. To demonstrate the effectiveness of the proposed method, several examples, including a VFO differintegrator, two pure VFO differentiators and a pure VFO integrator, are given in Section 3, in which the weighting function is adjusted by an iterative technique such that the peak absolute error of variable frequency response can be reduced as much as possible. Finally, the conclusions are given in Section 4

\section{Problem formulation}

For designing a VFO differintegrator, the desired response is given by

$D(\omega, p)=\mathrm{e}^{-\mathrm{j} l \omega}(\mathrm{j} \omega)^{p}, \quad p_{s} \leqslant p \leqslant p_{f}, \quad \omega_{s} \leqslant|\omega| \leqslant \omega_{f}$,

where $I$ is a prescribed delay and $p$ is the variable order of the designed differintegrator. If a pure VFO differentiator is designed, $p_{s} \geqslant 0$ and $\omega_{s} \geqslant 0$, while $p_{f} \leqslant 0$ and $\omega_{s}>0$ for designing a pure VFO integrator, and $p_{s}<0<p_{f}, \omega_{s}>0$ for the VFO differintegrator design. Let

$$
\begin{aligned}
\hat{D}(\omega, p) & =(\mathrm{j} \omega)^{p} \\
& =|\omega|^{p}\left[\cos \left(\frac{p \pi}{2}\right)+\mathrm{j} \operatorname{sgn}(\omega) \sin \left(\frac{p \pi}{2}\right)\right],
\end{aligned}
$$

where $\operatorname{sgn}(\cdot)$ is a sign function, then Eq. (1) can be represented by

$D(\omega, p)=\mathrm{e}^{-\mathrm{j} I \omega} \hat{D}(\omega, p)$.

For approximating the desired response, the used transfer function is characterized by

$H(z, p)=\sum_{n=0}^{N} h_{n}(p) z^{-n}$,

where the coefficients $h_{n}(p)$ are expressed as the polynomials of $p$

$h_{n}(p)=\sum_{m=0}^{M} h(n, m) p^{m}$,

hence Eq. (4) becomes

$H(z, p)=\sum_{n=0}^{N} \sum_{m=0}^{M} h(n, m) p^{m} z^{-n}$.

For simplicity, only even $N$ is used in this section and the case for odd $N$ will be given in Section 3 . According to the symmetric and antisymmetric characteristics for the real part and imaginary part of (2), respectively, with respective to $\omega$, the coefficients $h(n, m)$ in (6) are divided into even part and odd part by

$h(n, m)=h_{\mathrm{e}}(n, m)+h_{\mathrm{o}}(n, m)$, where

$$
\begin{aligned}
& h_{\mathrm{e}}\left(\frac{N}{2}+n, m\right)=\frac{1}{2}\left[h\left(\frac{N}{2}+n, m\right)+h\left(\frac{N}{2}-n, m\right)\right], \\
&-\frac{N}{2} \leqslant n \leqslant \frac{N}{2}, \quad 0 \leqslant m \leqslant M
\end{aligned}
$$

and

$$
\begin{aligned}
h_{\mathrm{o}}\left(\frac{N}{2}+n, m\right)= & \frac{1}{2}\left[h\left(\frac{N}{2}+n, m\right)-h\left(\frac{N}{2}-n, m\right)\right], \\
-\frac{N}{2} \leqslant n \leqslant \frac{N}{2}, & 0 \leqslant m \leqslant M .
\end{aligned}
$$

So, the frequency response of the designed filter can be formulated into

$$
\begin{aligned}
H\left(\mathrm{e}^{\mathrm{j} \omega}, p\right)= & \mathrm{e}^{-\mathrm{j}(N / 2) \omega}\left[\sum_{n=0}^{N / 2} \sum_{m=0}^{M} a(n, m) p^{m} \cos (n \omega)\right. \\
& \left.+\mathrm{j} \sum_{n=1}^{N / 2} \sum_{m=0}^{M} b(n, m) p^{m} \sin (n \omega)\right] \\
= & \mathrm{e}^{-\mathrm{j}(N / 2) \omega} \hat{H}(\omega, p),
\end{aligned}
$$

where

$a(n, m)= \begin{cases}h_{\mathrm{e}}\left(\frac{N}{2}, m\right), & n=0,0 \leqslant m \leqslant M, \\ 2 h_{\mathrm{e}}\left(\frac{N}{2}-n, m\right), & 1 \leqslant n \leqslant \frac{N}{2}, 0 \leqslant m \leqslant M,\end{cases}$

$b(n, m)=2 h_{\mathrm{o}}\left(\frac{N}{2}-n, m\right), \quad 1 \leqslant n \leqslant \frac{N}{2}, \quad 0 \leqslant m \leqslant M$

and

$$
\begin{aligned}
\hat{H}(\omega, p)= & \sum_{n=0}^{N / 2} \sum_{m=0}^{M} a(n, m) p^{m} \cos (n \omega) \\
& +\mathrm{j} \sum_{n=1}^{N / 2} \sum_{m=0}^{M} b(n, m) p^{m} \sin (n \omega) .
\end{aligned}
$$

Obviously, $I=N / 2$ in (1) and (3).

Let $\mathbf{A}$ and $\mathbf{B}$ be $(N / 2+1) \times(M+1)$ and $N / 2 \times(M+1)$ matrices defined by

$\mathbf{A}=\left[a(n, m), 0 \leqslant n \leqslant \frac{N}{2}, 0 \leqslant m \leqslant M\right]$

and

$\mathbf{B}=\left[b(n, m), 1 \leqslant n \leqslant \frac{N}{2}, 0 \leqslant m \leqslant M\right]$,

respectively; the following objective error function is used in the paper:

$$
\begin{aligned}
e(\mathbf{A}, \mathbf{B}) & =\sum_{i=0}^{K_{\omega}} \sum_{l=0}^{K_{p}} W\left(\omega_{i}\right)\left|D\left(\omega_{i}, p_{l}\right)-H\left(\mathrm{e}^{\mathrm{j} \omega_{i}}, p_{l}\right)\right|^{2} \\
& =\sum_{i=0}^{K_{\omega}} \sum_{l=0}^{K_{p}} W\left(\omega_{i}\right)\left|\hat{D}\left(\omega_{i}, p_{l}\right)-\hat{H}\left(\omega_{i}, p_{l}\right)\right|^{2}, \\
\omega_{i}= & \omega_{s}+\frac{i\left(\omega_{f}-\omega_{s}\right)}{K_{\omega}}, p_{l}=p_{s}+\frac{l\left(p_{f}-p_{s}\right)}{K_{p}},
\end{aligned}
$$

where a $\left(K_{\omega}+1\right) \times\left(K_{p}+1\right)$ grid is chosen for the error evaluation, and $W(\omega)$ is a positive weighting function. In 
the paper, $K_{\omega}=K_{p}=200$ is used. By Pythagorean law,

$e(\mathbf{A}, \mathbf{B})$

$$
\begin{aligned}
= & \sum_{i=0}^{K_{\omega}} \sum_{l=0}^{K_{p}} W\left(\omega_{i}\right)\left[\omega_{i}^{p_{l}} \cos \left(\frac{p_{l} \pi}{2}\right)-\sum_{n=0}^{N / 2} \sum_{m=0}^{M} a(n, m) p_{l}^{m} \cos \left(n \omega_{i}\right)\right]^{2} \\
& +\sum_{i=0}^{K_{\omega}} \sum_{l=0}^{K_{p}} W\left(\omega_{i}\right)\left[\omega_{i}^{p_{l}} \sin \left(\frac{p_{l} \pi}{2}\right)-\sum_{n=1}^{N / 2} \sum_{m=0}^{M} b(n, m) p_{l}^{m} .\right. \\
& \left.\times \sin \left(n \omega_{i}\right)\right]^{2} .
\end{aligned}
$$

Eq. (14) can be expressed in matrix form as

$$
\begin{aligned}
e(\mathbf{A}, \mathbf{B})= & \operatorname{tr}\left[\left(\mathbf{D}_{\mathbf{A}}-\mathbf{C} \mathbf{A} \mathbf{P}^{\mathrm{T}}\right)^{\mathrm{T}}\left(\mathbf{D}_{\mathbf{A}}-\mathbf{C A} \mathbf{P}^{\mathrm{T}}\right)\right] \\
& +\operatorname{tr}\left[\left(\mathbf{D}_{\mathbf{B}}-\mathbf{S B} \mathbf{P}^{\mathrm{T}}\right)^{\mathrm{T}}\left(\mathbf{D}_{\mathbf{B}}-\mathbf{S B P}^{\mathrm{T}}\right)\right] \\
= & e(\mathbf{A})+e(\mathbf{B}),
\end{aligned}
$$

where $\operatorname{tr}(\cdot)$ denotes a trace operator, the superscript $\mathrm{T}$ denotes a transpose operator,

$$
\begin{aligned}
& \mathbf{D}_{\mathbf{A}}=\left[W^{1 / 2}\left(\omega_{i}\right) \omega_{i}^{p_{l}} \cos \left(\frac{p_{l} \pi}{2}\right), 0 \leqslant i \leqslant K_{\omega}, 0 \leqslant l \leqslant K_{p}\right], \\
& \mathbf{D}_{\mathbf{B}}=\left[W^{1 / 2}\left(\omega_{i}\right) \omega_{i}^{p_{l}} \sin \left(\frac{p_{l} \pi}{2}\right), 0 \leqslant i \leqslant K_{\omega}, 0 \leqslant l \leqslant K_{p}\right], \\
& \mathbf{C}=\left[W^{1 / 2}\left(\omega_{i}\right) \cos \left(n \omega_{i}\right), 0 \leqslant i \leqslant K_{\omega}, 0 \leqslant n \leqslant \frac{N}{2}\right], \\
& \mathbf{S}=\left[W^{1 / 2}\left(\omega_{i}\right) \sin \left(n \omega_{i}\right), 0 \leqslant i \leqslant K_{\omega}, 1 \leqslant n \leqslant \frac{N}{2}\right], \\
& \mathbf{P}=\left[p_{l}^{m}, 0 \leqslant l \leqslant K_{p}, 0 \leqslant m \leqslant M\right]
\end{aligned}
$$

and

$$
\begin{aligned}
e(\mathbf{A}) & =\operatorname{tr}\left[\left(\mathbf{D}_{\mathbf{A}}-\mathbf{C} \mathbf{A} \mathbf{P}^{\mathrm{T}}\right)^{\mathrm{T}}\left(\mathbf{D}_{\mathbf{A}}-\mathbf{C} \mathbf{A} \mathbf{P}^{\mathrm{T}}\right)\right] \\
& =\operatorname{tr}\left[\mathbf{D}_{\mathbf{A}}^{\mathrm{T}} \mathbf{D}_{\mathbf{A}}-\mathbf{D}_{\mathbf{A}}^{\mathrm{T}} \mathbf{C} \mathbf{A} \mathbf{P}^{\mathrm{T}}-\left(\mathbf{C} \mathbf{A} \mathbf{P}^{\mathrm{T}}\right)^{\mathrm{T}} \mathbf{D}_{\mathbf{A}}+\left(\mathbf{C} \mathbf{A} \mathbf{P}^{\mathrm{T}}\right)^{\mathrm{T}}\left(\mathbf{C} \mathbf{A} \mathbf{P}^{\mathrm{T}}\right)\right],
\end{aligned}
$$

$$
\begin{aligned}
e(\mathbf{B}) & =\operatorname{tr}\left[\left(\mathbf{D}_{\mathbf{B}}-\mathbf{S B} \mathbf{P}^{\mathrm{T}}\right)^{\mathrm{T}}\left(\mathbf{D}_{\mathbf{B}}-\mathbf{S} \mathbf{B} \mathbf{P}^{\mathrm{T}}\right)\right] \\
& =\operatorname{tr}\left[\mathbf{D}_{\mathbf{B}}^{\mathrm{T}} \mathbf{D}_{\mathbf{B}}-\mathbf{D}_{\mathbf{B}}^{\mathrm{T}} \mathbf{S B} \mathbf{P}^{\mathrm{T}}-\left(\mathbf{S B} \mathbf{P}^{\mathrm{T}}\right)^{\mathrm{T}} \mathbf{D}_{\mathbf{B}}+\left(\mathbf{S} \mathbf{B} \mathbf{P}^{\mathrm{T}}\right)^{\mathrm{T}}\left(\mathbf{S B P} \mathbf{P}^{\mathrm{T}}\right)\right] .
\end{aligned}
$$

Differentiating $e(\mathbf{A}, \mathbf{B})$ with respect to $\mathbf{A}[35]$,

$$
\begin{aligned}
\frac{\partial e(\mathbf{A}, \mathbf{B})}{\partial \mathbf{A}}= & \frac{\partial e(\mathbf{A})}{\partial \mathbf{A}}=-\left(\mathbf{D}_{A}^{\mathrm{T}} \mathbf{C}\right)^{\mathrm{T}}\left(\mathbf{P}^{\mathrm{T}}\right)^{\mathrm{T}}-\mathbf{C}^{\mathrm{T}} \mathbf{D}_{\mathbf{A}} \mathbf{P} \\
& +\left(\mathbf{P} \mathbf{A}^{\mathrm{T}} \mathbf{C}^{\mathrm{T}} \mathbf{C}\right)^{\mathrm{T}}\left(\mathbf{P}^{\mathrm{T}}\right)^{\mathrm{T}}+\mathbf{C}^{\mathrm{T}} \mathbf{C} \mathbf{A} \mathbf{P}^{\mathrm{T}} \mathbf{P},
\end{aligned}
$$

which is then set to zero, and the coefficient matrix $\mathbf{A}$ can be obtained as

$$
\mathbf{A}=\left(\mathbf{C}^{\mathrm{T}} \mathbf{C}\right)^{-1} \mathbf{C}^{\mathrm{T}} \mathbf{D}_{\mathbf{A}} \mathbf{P}\left(\mathbf{P}^{\mathrm{T}} \mathbf{P}\right)^{-1} \text {. }
$$

Similarly, the coefficient matrix $\mathbf{B}$ can be achieved by differentiating $e(\mathbf{A}, \mathbf{B})$ with respect to $\mathbf{B}$ and setting the result to zero, which yields

$$
\mathbf{B}=\left(\mathbf{S}^{\mathrm{T}} \mathbf{S}\right)^{-1} \mathbf{S}^{\mathrm{T}} \mathbf{D}_{\mathbf{B}} \mathbf{P}\left(\mathbf{P}^{\mathrm{T}} \mathbf{P}\right)^{-1} .
$$

Notice that the weighting function $W(\omega)$ has been incorporated in the relevant matrices, so that the peak absolute error of variable frequency response can be reduced by a proper iterative method, which will be shown in Section 3.

\section{Numerical examples and discussions}

To demonstrate the effectiveness and flexibility of the proposed method, several examples including a VFO differintegrator, two pure VFO differentiators and a pure VFO integrator are presented in this section. To evaluate the performance, the normalized root-mean-squared error of variable frequency response and the maximum absolute error of variable frequency response are defined by

$\varepsilon_{r m s}=\left[\frac{\int_{p_{s}}^{p_{f}} \int_{\omega_{s}}^{\omega_{f}}\left|D(\omega, p)-H\left(\mathrm{e}^{\mathrm{j} \omega}, p\right)\right|^{2} \mathrm{~d} \omega \mathrm{d} p}{\int_{p_{s}}^{p_{f}} \int_{\omega_{s}}^{\omega_{f}}|D(\omega, p)|^{2} \mathrm{~d} \omega \mathrm{d} p}\right]^{1 / 2} \times 100 \%$

and

$\varepsilon_{m}=\max \left\{\left|D(\omega, p)-H\left(\mathrm{e}^{\mathrm{j} \omega}, p\right)\right|, \omega_{s} \leqslant \omega \leqslant \omega_{p}, p_{s} \leqslant p \leqslant p_{f}\right\}$,

respectively. To compute the error $\varepsilon_{r m s}$, the general trapezoidal rule is used [34] with step sizes $\left(\omega_{f}-\omega_{s}\right) / 200$ and $\left(p_{f}-p_{s}\right) / 200$ for $\omega$-axis and $p$-axis, respectively. Also, the error $\varepsilon_{m}$ is computed with the same sampling sizes as above.

Example 1. This example deals with the least-squares design of a VFO differintegrator with $N=40, M=5$, $\omega_{s}=(0.05) \pi, \quad \omega_{f}=(0.95) \pi, \quad p_{s}=-0.5, \quad p_{f}=0.5 \quad$ and $W(\omega)=1$. Fig. 1(a) and (b) present the obtained magnitude response and the absolute error of variable frequency response, respectively, and the errors $\varepsilon_{r m s} \approx 0.60277728 \%$ and $\varepsilon_{m}=0.1369375$.

It is noted that the phase difference between $\omega=\pi$ and $\omega=-\pi$ is $p \pi$, which is not an integer multiple of $2 \pi$ for all $p$ in the range $\left[p_{s}, p_{f}\right]$, so it is not recommended to set $\omega_{f}=\pi$. However, for comparing with the results of [22], the differintegrator is designed again with $\omega_{s}=(0.01) \pi$, $\omega_{f}=\pi$. If the computation of integration in [22] is implemented by using the trapezoidal rule with step sizes $\left(\omega_{f}-\omega_{s}\right) / 200$ and $\left(p_{f}-p_{s}\right) / 200$ for $\omega$-axis and $p$-axis, respectively, both the method of [22] and the proposed method induce the exactly same results: $\varepsilon_{r m s} \approx 10.01497046 \%$ and $\varepsilon_{m}=3.11763459$.

Example 2. For designing a pure VFO differentiator, $0 \leqslant p_{s}<p_{f}$. For example, a VFO differentiator is designed with $N=30, M=6, \omega_{s}=0, \omega_{f}=0.9 \pi, p_{s}=1, p_{f}=2$ and $W(\omega)=1$, the variable magnitude response and the absolute error of variable frequency response are shown in Fig. 2(a) and (b), respectively, and the errors $\varepsilon_{r m s} \approx 0.166372 \%$ and $\varepsilon_{m}=0.03382684$ which are better than $\varepsilon_{r m s} \approx 1.17212338 \%$ and $\varepsilon_{m}=0.11866149$ obtained with the method of [22] where the ill-conditioned problem will occur for the relevant matrix.

To further reduce the maximum absolute error of variable frequency response, $\varepsilon_{m}$, the iterative method in $[32,33]$ is modified and applied as follows. Before 

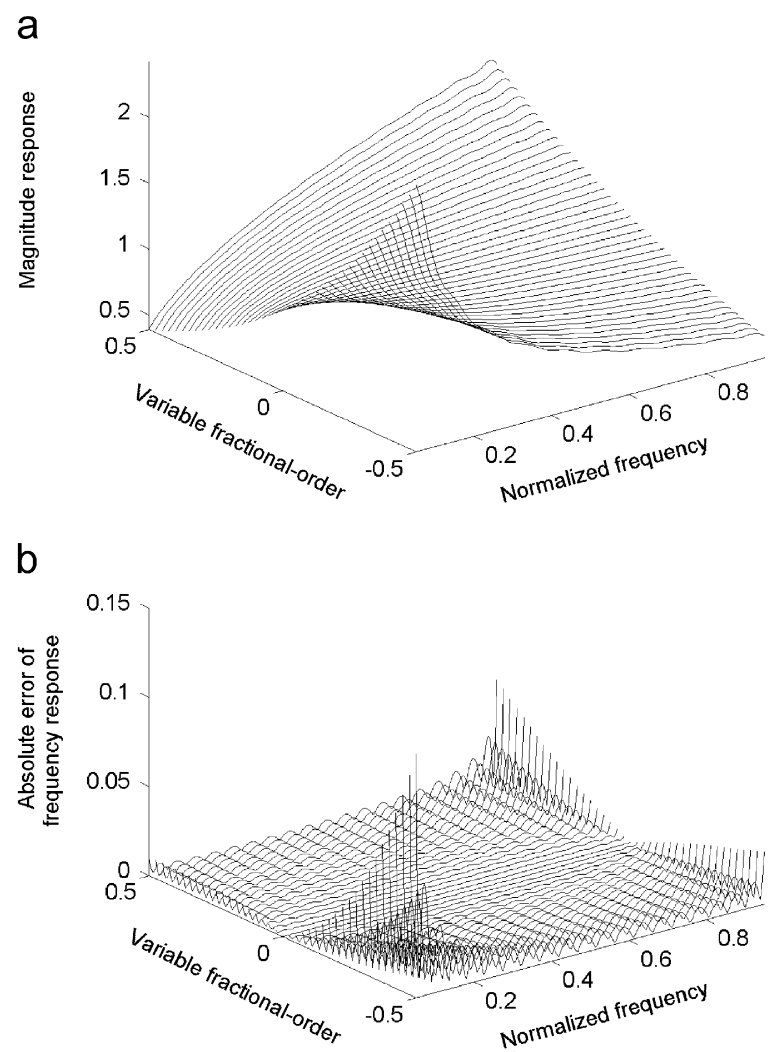

Fig. 1. Design of a VFO differintegrator with $N=40, M=5, \omega_{s}=(0.05) \pi$, $\omega_{f}=(0.95) \pi, p_{s}=-0.5$ and $p_{f}=0.5$. (a) Variable magnitude response. (b) Absolute error of variable frequency response.

describing the modified algorithm, some notations are defined as below:

$E(\omega, p)$ : the absolute error function defined by $E(\omega, p)=\left|D(\omega, p)-H\left(\mathrm{e}^{\mathrm{j} \omega}, p\right)\right|$,

$p_{m}$ : the variable $p$ where the maximum of $E(\omega, p)$ occurs for the first iteration,

$\gamma_{i}$ : the $i$ th absolute error ripple of $E\left(\omega, p_{m}\right)$ with ripple interval $\left(\hat{\omega}_{i-1}, \hat{\omega}_{i}\right]$ (except that the first ripple interval is $\left.\left[\omega_{s}, \hat{\omega}_{1}\right]\right)$,

$\delta: \max \left\{\gamma_{i}\right\}$,

$\rho: \min \left\{\gamma_{i}\right\}$.

The proposed iterative method is described in detail as below.

Step 1: Initiate the weighting function

$W(\omega)=1, \quad \omega_{s} \leqslant \omega \leqslant \omega_{f}$.

Step 2: Find the coefficient matrices $\mathbf{A}$ and $\mathbf{B}$ by (20) and (21), respectively.

Step 3: Find $p_{m}$ for the first iteration only, and search for $\gamma_{i}, \delta$ and $\rho$ for all iterations.

Step 4: Check whether the error $E\left(\omega, p_{m}\right)$ is nearly equiripple by

$\delta_{\rho}=\frac{\delta-\rho}{\delta} \leqslant \varepsilon$, where $\delta_{\rho}$ is the relative peak error ratio and $\varepsilon$ is a preassigned very small positive constant. If the condition is satisfied, stop the process; otherwise go to the next step.

Step 5: Compute the unnormalized weighting function

$\hat{W}(\omega)=\left\{\begin{array}{ll}W(\omega) \gamma_{i}^{2}, & i=1, \quad \omega_{s} \leqslant \omega \leqslant \hat{\omega}_{1} \\ W(\omega) \gamma_{i}^{2}, & 2 \leqslant i \leqslant \hat{I}, \quad \hat{\omega}_{i-1}<\omega \leqslant \hat{\omega}_{i}\end{array}\right.$,

where $\hat{I}$ is the number of ripples in $\left[\omega_{s}, \omega_{f}\right]$, and find its maximum value

$\delta_{W}=\max \left\{\hat{W}(\omega), \omega_{s} \leqslant \omega \leqslant \omega_{f}\right\}$.

Then update the weighting function by

$W(\omega)=\frac{\hat{W}(\omega)}{\delta_{W}}, \quad \omega_{s} \leqslant \omega \leqslant \omega_{f}$

and go to Step 2 .

For example, if the specification of the designed VFO differentiator is the same as that stated above, and $\varepsilon=0.01$ is used, the design takes seven iterations; the absolute error of variable frequency responses is shown in Fig. 2(c) and $\varepsilon_{r m s} \approx 0.27842619 \%, \varepsilon_{m}=0.01215898$. To clearly show the difference between the results of the first and the seventh iterations, the error curves $E\left(\omega, p_{m}\right)$ are illustrated in Fig. 2(d). Also, the final weighting function is illustrated in Fig. 2(e).

Example 3. A pure VFO integrator is designed with $N=60, M=6, \omega_{s}=(0.05) \pi, \omega_{f}=(0.9) \pi, p_{s}=-1.5$, and $p_{f}=-0.5$ in this example. First, a least-squares design is presented, and the variable magnitude response and the absolute error of variable frequency response are shown in Fig. 3(a) and (b), respectively, where $\varepsilon_{r m s} \approx 1.3779794 \%$ and $\varepsilon_{m}=0.33681498$. By using the method in [22], it yields $\varepsilon_{r m s} \approx 3.29702665 \%$ and $\varepsilon_{m}=0.34745782$, which shows that the proposed method is better for the design. Also, the iterative method shown in Example 2 can be applied here; the absolute error of variable frequency response after five iterations is shown in Fig. 3(c) if $\varepsilon=0.01$ is used where $\varepsilon_{r m s} \approx 3.05425137 \%$ and $\varepsilon_{m}=0.13889478$ and the error curves $E\left(\omega, p_{m}\right)$ in the first and last iterations are presented in Fig. 3(d).

Example 4. As to the design for odd $N$, the transfer function becomes

$$
\begin{aligned}
H(z, p) & =\sum_{n=0}^{N} \sum_{m=0}^{M} h(n, m) p^{m} z^{-n} \\
& =\sum_{m=0}^{M}\left\{\sum_{n=0}^{N}\left[h_{\mathrm{e}}(n, m)+h_{0}(n, m)\right] z^{-n}\right\} p^{m},
\end{aligned}
$$

where

$$
\begin{aligned}
h_{\mathrm{e}}\left(\frac{N+1}{2}-n, m\right) & =h_{\mathrm{e}}\left(\frac{N-1}{2}+n, m\right) \\
& =\frac{1}{2}\left[h\left(\frac{N+1}{2}-n, m\right)+h\left(\frac{N-1}{2}+n, m\right)\right], \\
1 \leqslant n \leqslant \frac{N+1}{2}, & 0 \leqslant m \leqslant M
\end{aligned}
$$


a

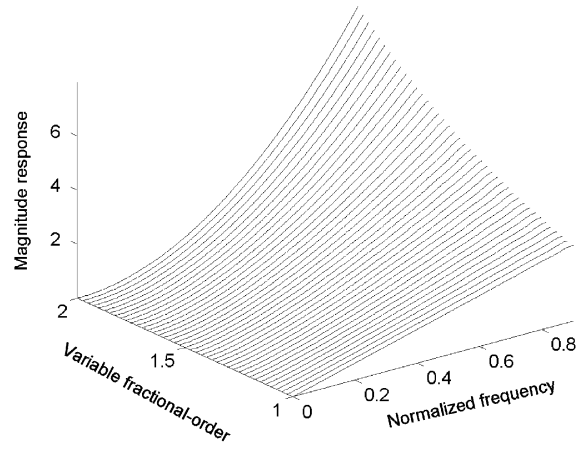

C

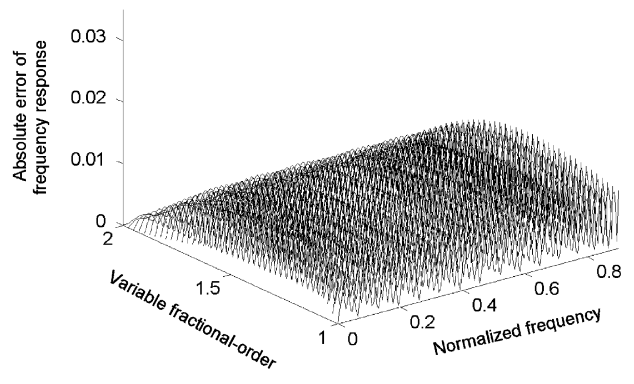

e b

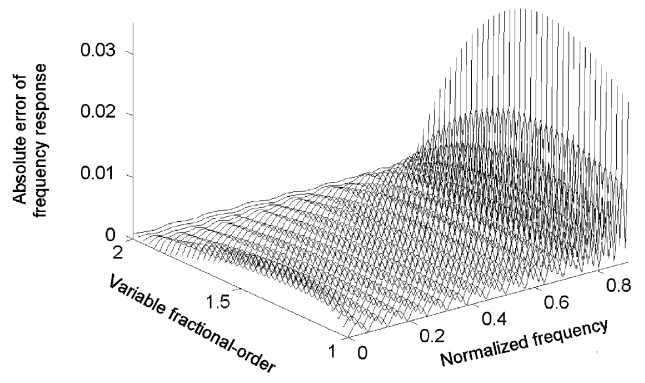

d

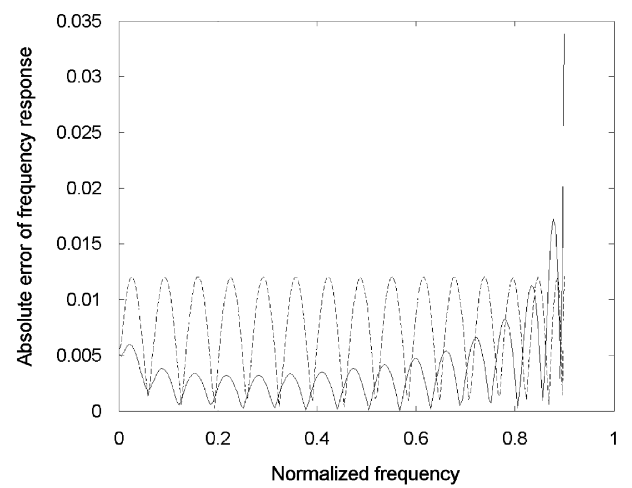

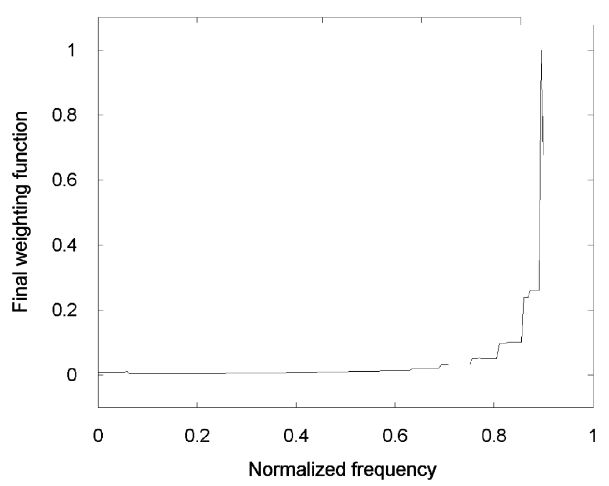

Fig. 2. Design of a VFO differentiator with $N=30, M=6, \omega_{s}=0, \omega_{f}=(0.9) \pi, p_{s}=1$ and $p_{f}=2$. (a) Variable magnitude response. (b) Absolute error of variable frequency response (least-squares design). (c) Absolute error of variable frequency response (iterative design). (d) Absolute errors $E\left(\omega, p_{m}\right)$ (solid line: first iteration, dotted line: seventh iteration). (e) Final weighting function.

and

$$
\begin{aligned}
h_{\mathrm{o}}\left(\frac{N+1}{2}-n, m\right) & =-h_{\mathrm{o}}\left(\frac{N-1}{2}+n, m\right) \\
& =\frac{1}{2}\left[h\left(\frac{N+1}{2}-n, m\right)-h\left(\frac{N-1}{2}+n, m\right)\right], \\
1 \leqslant n \leqslant \frac{N+1}{2}, & 0 \leqslant m \leqslant M,
\end{aligned}
$$

and its frequency response can be formulated into

$$
\begin{aligned}
H\left(\mathrm{e}^{\mathrm{j} \omega}, p\right)= & \mathrm{e}^{-\mathrm{j}(N / 2) \omega}\left[\sum_{n=1}^{(N+1) / 2} \sum_{m=0}^{M} a(n, m) p^{m} \cos \left(\left(n-\frac{1}{2}\right) \omega\right)\right. \\
& \left.+\mathrm{j} \sum_{n=1}^{(N+1) / 2} \sum_{m=0}^{M} b(n, m) p^{m} \sin \left(\left(n-\frac{1}{2}\right) \omega\right)\right], \quad
\end{aligned}
$$

where

$$
a(n, m)=2 h_{\mathrm{e}}\left(\frac{N+1}{2}-n, m\right), \quad 1 \leqslant n \leqslant \frac{N+1}{2}, 0 \leqslant m \leqslant M
$$

and

$b(n, m)=2 h_{0}\left(\frac{N+1}{2}-n, m\right), \quad 1 \leqslant n \leqslant \frac{N+1}{2}, 0 \leqslant m \leqslant M$.

So the technique described in Section 2 can also be applied to design VFO FIR differintegrators with odd $N$. For example, when $N=31, M=6, \omega_{s}=0, \omega_{f}=0.9 \pi, p_{s}=1$, $p_{f}=2$ and $W(\omega)=1$, the absolute error of variable frequency response is shown in Fig. 4(a), and 
a

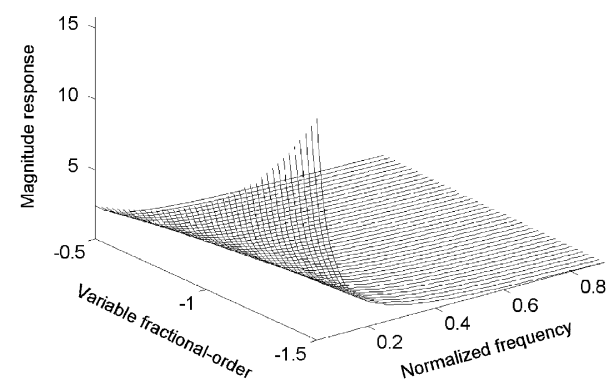

C

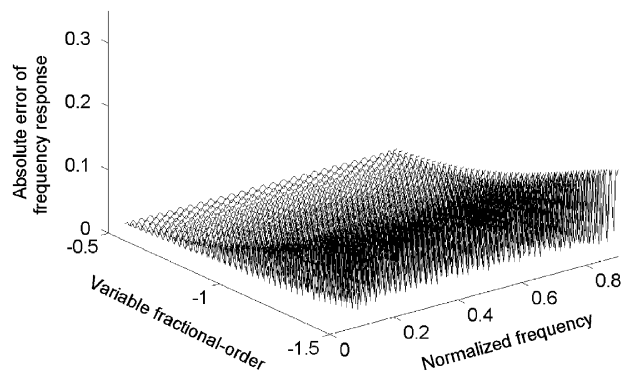

b

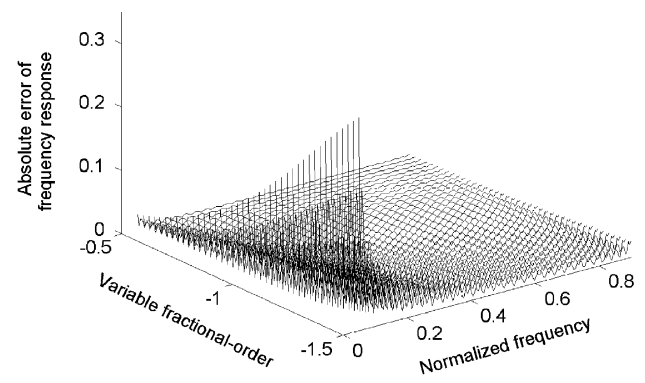

d

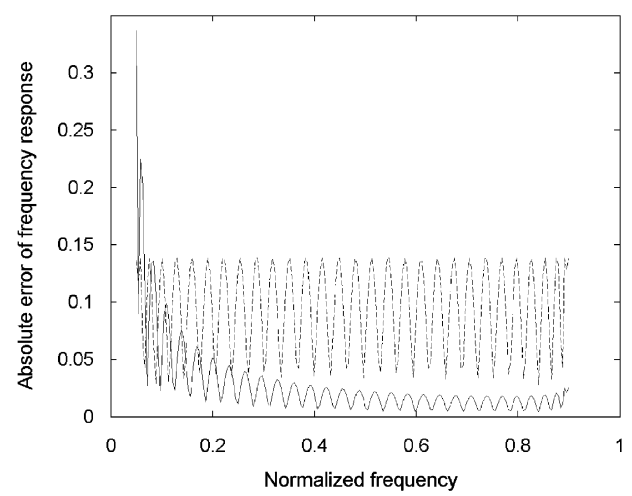

Fig. 3. Design of a VFO integrator with $N=60, M=6, \omega_{s}=(0.05) \pi, \omega_{f}=(0.9) \pi, p_{s}=-1.5$ and $p_{f}=-0.5$. (a) Variable magnitude response. (b) Absolute error of variable frequency response (least-squares design). (c) Absolute error of variable frequency response (iterative design). (d) Absolute error $E\left(\omega, p_{m}\right)$ (solid line: first iteration, dotted line: fifth iteration).

a

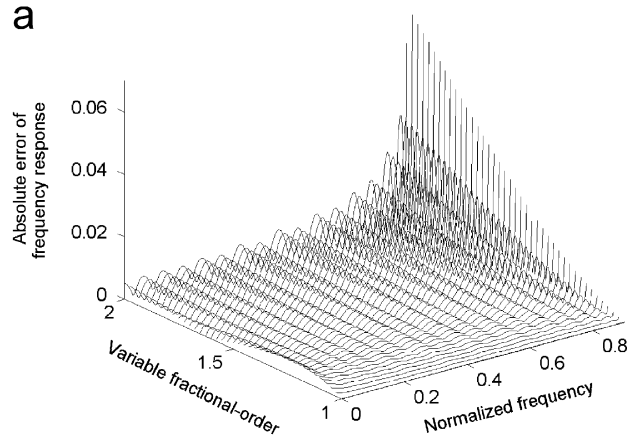

b

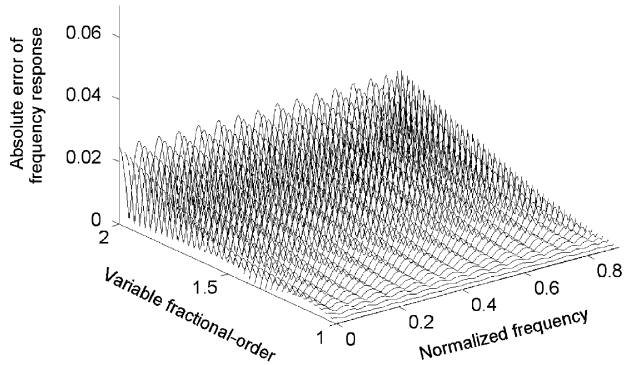

Fig. 4. Design of a VFO differentiator with $N=31, M=6, \omega_{s}=0, \omega_{f}=(0.9) \pi, p_{s}=1$ and $p_{f}=2$. (a) Absolute error of variable frequency response (leastsquares design). (b) Absolute error of variable frequency response (iterative design).

$\varepsilon_{r m s} \approx 0.21197558 \%, \varepsilon_{m}=0.06927072$ which are also smaller than those obtained with the method of [22] where $\varepsilon_{r m s} \approx 1.16104267 \%$ and $\varepsilon_{m}=0.16102323$. Also, if the iterative process in Example 2 is applied here with $\varepsilon=0.01$, the process stops after the ninth iteration and the absolute error of variable frequency response is presented in Fig. 4(b) where $\varepsilon_{r m s} \approx 0.36685739 \%$ and $\varepsilon_{m}=0.02494169$.

Basing on the presented examples above, there are some issues which can be further discussed.

a. During the iterative process, the relative peak error ratio $\delta_{\rho}$ will reduce gradually, and the process can stop when it is small enough which means the specified error curve is almost equiripple. For example, the trace of the relative peak error ratio in Example 3 is illustrated in Fig. 5 in which the ratio reduces to 0.00142864 in the eighth iteration and then varies between 0.001 and 0.002 .

b. Comparing with the existing weighted-least-squares approach such as the method in [22] which is also widely used to design variable fractional-delay FIR digital filters, the proposed method generally can get better results for the design of pure VFO differentiators and pure VFO integrators as shown in Examples 2-4 


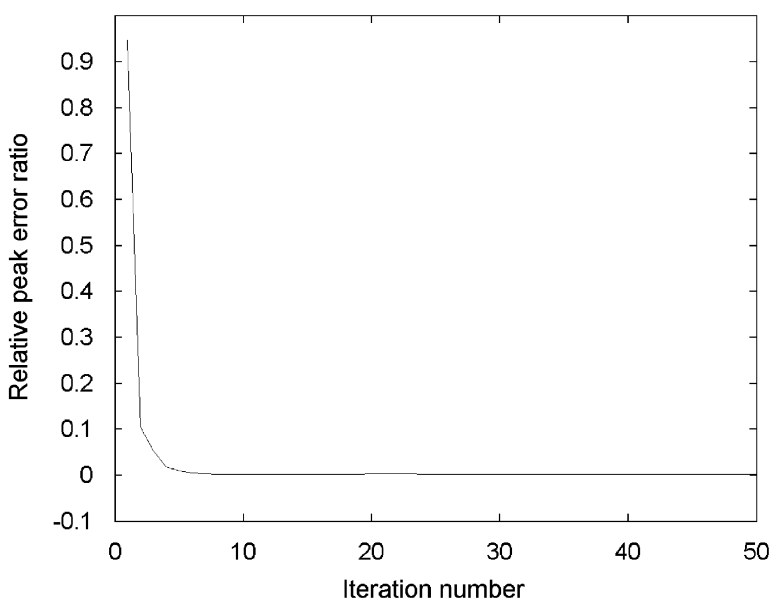

Fig. 5. Trace of the relative peak error ratio in Example 3.

due to the ill-conditioned problem may occur for the former method.

c. Generally, a 2-D weighting function $W\left(\omega_{1}, \omega_{2}\right)$ can be used for the weighted-least-squares design of 2-D digital filters. But for the design of VFO differintegrators, there is no an explicit way so far to find a proper 2-D weighting function $W(\omega, p)$ to adjust effectively the related error, especially for the $p$-axis. The analogous case also occurs for the design of variable fractional-delay FIR filters [28]. From several experiments, when the weighting function is updated dependent on the parameter $p$ as well as $\omega$. It generally does not yield better result. So, we only use the $1-D$ weighting function in the paper. Although the minimax design for all $p$ cannot be obtained, but the peak absolute error of variable frequency response has been minimized effectively as shown in Examples 2-4.

d. In the paper, a $\left(K_{\omega}+1\right) \times\left(K_{p}+1\right)=201 \times 201$ grid is used for the error evaluation in (13) and the obtained results are satisfactory throughout our experiments. Although higher density of grid points can be used, but it does not guarantee a better result. For example, when $K_{\omega}=400$ is used in Example 3, the design stops after nine iterations and $\varepsilon_{r m s} \approx 3.08547182 \%$, $\varepsilon_{m}=0.14133676$ which are not so good as the results in Example 3.

\section{Conclusions}

In the paper, a new method has been proposed for the design of VFO FIR differintegrators in weighted-leastsquares sense. Also, an iterative method is presented, so that the weighting function can be adjusted and the peak absolute error of variable frequency response can be reduced as much as possible. Several experiments show that the convergence of the iterative process is satisfactory. To demonstrate the effectiveness of the proposed method, several design examples, including a VFO differintegrator, two pure VFO differentiators and a pure
VFO integrator, are presented. Obviously, the method can also be extended for the design of other variable digital filters.

\section{References}

[1] K.B. Oldham, J. Spanier, The Fractional Calculus, Academic Press, New York, 1974

[2] K.S. Miller, B. Ross, An Introduction to the Fractional Calculus and Functional Differential Equations, Wiley, New York, 1993.

[3] K. Ogata, Discrete-time Control Systems, Prentice-Hall, Englewood Cliffs, NJ, 1987.

[4] I. Podlubny, Fractional Differential Equations, Academic Press, San Diego, CA, 1999.

[5] B. Mbodje, G. Montseny, Boundary fractional derivative control of the wave equation, IEEE Trans. Automat. Control 40 (2) (1995) 378-382.

[6] N. Engheta, On fractional calculus and fractional multipoles in electromagnetism, IEEE Trans. Antennas Propag. 44 (4) (1996) 554-566.

[7] R. Hilfer (Ed.), Application of Fractional Calculus in Physics, World Scientific, River Edge, NJ, 2000.

[8] B.M. Vinagre, I. Podlubny, A. Hernandez, V. Feliu, Some approximation of fractional order operators used in control theory and applications, J. Fractional Calculus Appl. Anal. 4 (2001) 47-66.

[9] B. Mathieu, P. Melchior, A. Ousaloup, C. Ceyral, Fractional differentiation for edge detection, Signal Processing 83 (2003) 2421-2432.

[10] Y.Q. Chen, B.M. Vinagre, I. Podlubny, Continued fraction expansion approaches to discretizing fractional order derivatives-an expository review, Nonlinear Dynamics 38 (2004) 155-170.

[11] C.-C. Tseng, Design of fractional order digital FIR differentiators, IEEE Signal Process. Lett. 8 (3) (2001) 77-79.

[12] Y.Q. Chen, K.L. Moore, Discretization schemes for fractional-order differentiators and integrators, IEEE Trans. Circuits Systems I 49 (3) (2002) 363-367.

[13] Y.Q. Chen, B.M. Vinagre, A new IIR-type digital fractional order differentiator, Signal Processing 83 (2003) 2359-2365.

[14] P. Ostalczyk, Fundamental properties of the fractional-order discrete-time integrator, Signal Processing 83 (2003) 2367-2376.

[15] S. Samadi, M.O. Ahmad, M.N.S. Swamy, Exact fractional-order differentiators for polynomial signals, IEEE Signal Process. Lett. 11 (6) (2004) 529-532.

[16] H. Zhao, G. Qin, L. Yao, J. Yu, Design of fractional order digital FIR differentiators using frequency response approximation, in: Proceedings of the 2005 International Conference on Communications, Circuits and Systems, May 2005, pp. 1318-1321.

[17] C.-C. Tseng, Improved design of digital fractional-order differentiators using fractional sample delay, IEEE Trans. Circuits Systems I 53 (1) (2006) 193-203.

[18] G. Maione, Concerning continued fractions representation of noninteger order digital differentiators, IEEE Signal Process. Lett. 13 (12) (2006) 725-728

[19] C.-C. Tseng, Design of FIR and IIR fractional order Simpson digital integrators, Signal Processing 87 (2007) 1045-1057.

[20] C.K.S. Pun, S.C. Chan, K.S. Yeung, K.L. Ho, On the design and implementation of FIR and IIR digital filters with variable frequency characteristics, IEEE Trans. Circuits Systems II 49 (11) (2002) 689-703.

[21] C.-C. Tseng, Design and application of variable fractional order differentiator, in: The 2004 IEEE Asia-Pacific Conference on Circuits and Systems, December 2004, pp. 405-408.

[22] C.-C. Tseng, Design of variable and adaptive fractional order FIR differentiators, Signal Processing 86 (2006) 2554-2566.

[23] T.-B. Deng, Design of arbitrary-phase variable digital filters using SVD-based vector-array decomposition, IEEE Trans. Circuits Systems I 52 (1) (2005) 148-167.

[24] H.H. Dam, A. Cantoni, K.L. Teo, S. Nordholm, Variable digital filter with least-square criterion and peak gain constraints, IEEE Trans. Circuits Systems II 54 (1) (2007) 24-28.

[25] T.I. Laakso, V. Valimaki, M. Karjalainen, U.K. Laine, Splitting the unit delay: tools for fractional delay filter design, IEEE Signal Process. Mag. 13 (1996) 30-60.

[26] C.W. Farrow, A continuously variable digital delay elements, in: Proceedings of the IEEE International Symposium on Circuits Systems, June 1988, pp. 2641-2645. 
[27] J.-C. Liu, S.-J. You, Weighted least squares near-equiripple approximation of variable fractional delay FIR filters, IET Signal Process. 1 (2007) 66-72.

[28] T.-B. Deng, Y. Lian, Weighted-least-squares design of variable fractional-delay FIR filters using coefficient symmetry, IEEE Trans. Signal Process. 54 (8) (2006) 3023-3038.

[29] T.-B. Deng, Symmetric structures for odd-order maximally flat and weighted-least-squares variable fractionaldelay filters, IEEE Trans. Circuits Systems I 54 (12) (2007) 2718-2732.

[30] M.O. Ahmad, J.-D. Wang, An analytical least-square solution to the design problem of two-dimensional FIR filters with quadrantically symmetric or antisymmetric frequency response, IEEE Trans. Circuits Systems 36 (7) (1989) 968-979.
[31] S.-C. Pei, J.-J. Shyu, General form for designing two-dimensional quadrantally symmetric linear-phase FIR digital filters by analytical least-squares method, Signal Processing 48 (1996) 165-174.

[32] S.-C. Pei, J.-J. Shyu, Fast design of 2-D linear-phase complex FIR digital filters by analytical least squares method, IEEE Trans. Signal Process. 44 (12) (1996) 3157-3161.

[33] C.Y. Chi, Y.T. Kou, A new self-initiated optimum WLS approximation method for the design of linear phase FIR digital filters, in: Proceedings of the IEEE International Symposium on Circuits and Systems, June 1991, pp. 168-171.

[34] K.E. Atkinson, An Introduction to Numerical Analysis, second ed, Wiley, New York, 1989.

[35] K.M. Abadir, J.R. Magnus, Matrix Algebra, Cambridge University Press, Cambridge, 2005. 